\title{
Can we measure memes?
}

\author{
Adam McNamara* \\ Department of Psychology, University of Surrey, Surrey, UK
}

Edited by:

Lisa M. Renzi, The University of Georgia, USA

\section{Reviewed by:}

Robert Finkelstein, Robotic Technology

Inc., USA

Francis Heylighen, Vrije Universiteit

Brussel, Belgium

\section{*Correspondence}

Adam McNamara, Department of

Psychology, University of Surrey,

Guildford, Surrey, GU2 7XH, UK.

e-mail:a.mcnamara@surrey.ac.uk
Memes are the fundamental unit of cultural evolution and have been left upon the periphery of cognitive neuroscience due to their inexact definition and the consequent presumption that they are impossible to measure. Here it is argued that although a precise definition of memes is rather difficult it does not preclude highly controlled experiments studying the neural substrates of their initiation and replication. In this paper, memes are termed as either internally or externally represented (i-memes/e-memes) in relation to whether they are represented as a neural substrate within the central nervous system or in some other form within our environment. It is argued that neuroimaging technology is now sufficiently advanced to image the connectivity profiles of i-memes and critically, to measure changes to i-memes over time, i.e., as they evolve. It is argued that it is wrong to simply pass off memes as an alternative term for "stimulus" and "learnt associations" as it does not accurately account for the way in which natural stimuli may dynamically "evolve" as clearly observed in our cultural lives.

Keywords: meme, mirror neurons, fMRI, evolution, functional connectivity

\section{INTRODUCTION}

\section{MEMES, MIRROR NEURONS, AND MODERN NEUROIMAGING}

It has been suggested that the sudden explosion of what is considered fundamentally human: consciousness, culture, language, and intellect is a consequence of our evolved capacity to imitate (Donald, 1993). That the driving force for this cultural explosion was the generation of a second environ-mental space in which memes drove biological selection as well as genes (Blackmore, 1999). A meme is a replicator, a cultural unit operating under Darwinian evolutionary principles analogous to a gene, but a distinct replicator in its own right (Dawkins, 1976; Goodenough and Dawkins, 1994). A meme in layman terms is a concept or idea, embodied by a word, a phrase, a riff, image, or gesture. A meme "exists" in the world of ideas and replicates by imitation. Memes are fluffy, non-delineated concepts rendering them somewhat untouchable by cognitive neuroscience albeit that they share considerable functional overlap with a seminal discovery in neuroscience. Over a decade ago mirror neurons, encoding the intention of "others," were discovered (Gallese et al., 1996; Rizzolatti et al., 1996). It was quickly proposed that these neurons, located in regions highly involved in imitation, were the neural substrates upon which language could have evolved (Rizzolatti and Arbib, 1998). The biological observation of neurons facilitating action recognition and replication fully supported a theory of memetics (Blackmore, 2005) and the field of memetics has grown (Heylighen and Chielens, 2008). However cognitive neuroscience has given the idea a fairly wide birth, probably due to the aforementioned non-measurable, indefinable nature of memes. This paper briefly reviews the biologically based work on the evolution of language. The paper concludes cognitive neuroscience can, and should, apply considerable weight to the investigation of memetics. Firstly, the paper suggests that although memes may not be quantifiable, the traces of the neural processes involved in memetic replication and storage within the central nervous system (CNS) are measurable. Producing artificial memes for study is easily achieved and has already been done within experimental protocols in a number of fields (see Do We Measure Memes Already?). Modern neuroimaging techniques enable us to quantify changes in neural-networkfunctional-connectivity-profiles as we perceive, learn, memorize, imitate, or perhaps more accurately "replicate" memes.

\section{THE NEAT VIEW OF GENES}

According to popular imagination, genes fit nicely into the physical world view that we can imagine even if not see. They are encoded using a four letter alphabet, lie in long lines and one can imagine reading them in a sensible manner. They are easily defined and their function is delineated. Unfortunately for geneticists the truth is not as simple as the popular imagination would have one believe. Defining a gene/cistron is no easy task - let alone identifying a gene's impact on an organism's internal functions. Admittedly there are "start" and "end" coding sequences to a gene, but not all the sequence between these markers are read, non-coding intron sequences are scattered across the gene (Gilbert, 1978). Genes can overlap on the chromosome (Normark et al., 1983; Veeramachaneni et al., 2004; Sanna et al., 2008), differing genes can be coded upon the same strand, or not, within the same frame, or not. Overlap can affect regulation of gene expression at the level of transcription, mRNA processing, splicing, or translation (Boi et al., 2004). Introns often also lead to alternative RNA splicing making genes difficult to define by creating alternative pathways for protein expression (Berget et al., 1977; Breathnach et al., 1977). Alternative splicing can account for massive consequences on function (Cavara and Hollmann, 2008) and is crucial to many genes involved in generating immunity (Lynch, 2004). Taking into account the cellular environment, functionality of the "start," "end," "enhancing," and "silencing" sequences is entirely dependent upon other factors. The end product of genes is of course proteins, of which many of these protein's function is ultimately defined by its molecular environment. The action of a gene becomes far less clearly delineated when one moves away from the common man's overly deterministic 
concept of a gene and faces the real complexity that geneticists have to disentangle. It is estimated that we have identified less than $0.3 \%$ of all 650,000 estimated protein interactions among the $\sim 25,000$ human proteins (Stumpf et al., 2008). Genes replicate in the physical world and we can measure them. However, we began to measure genes via their phenotypes long before identifying where, and on which chromosome they resided. Genetics was born from the painstaking and careful observation of clear, single gene phenotypes in pea plant petal color (Mendel, 1951). In reality how a gene fits into the organization of an organism is not as clear as one may imagine, how a gene impacts on cellular events and ultimately an organism's phenotype is only, in very rare cases, fully known. Mendel did not discover the fundamentals of genetics by throwing his hands in the air and exclaiming that it was all too complex and immeasurable. It appears that this is the current scientific stance on memes.

\section{REPLICATION OF BEHAVIOR FROM A COGNITIVE NEUROSCIENCE PERSPECTIVE}

In the field of cognitive neuroscience imitation is recognized as a fundamental human skill (Arbib, 2005; Roth and Dicke, 2005; Iriki, 2006). Neurons have been identified which match action perception with action execution, as well as action recognition in the monkey (Gallese et al., 1996; Rizzolatti and Arbib, 1998). These neurons, coined "mirror neurons," were found in the ventralpre-motor region F5 and parietal region F7 and have since been intensively studied (Kohler et al., 2002; Raos et al., 2004, 2006; Ferrari et al., 2005). It appears that monkeys infer the "intention" of others' actions within their own motor systems (Umiltà et al., 2001). Significantly, the motor system ceased to be a region considered to have low level function somewhat like the strings of a puppeteer. It became recognized the motor system had an important role in the evolution of communicative skills and understanding. Human homologs to these regions have been proposed based on a body of converging evidence (Rizzolatti and Arbib, 1998; Binkofski and Buccino, 2006). There is little contesting the idea that ventralpre-motor area Brodmann Area 44 (BA44) is the human homolog to monkey region $\mathrm{F} 5$ and that the infero-parietal lobule constitutes the human homolog to monkey region F7. This fronto-parietal network has been demonstrated as involved in complex hand gestures and movements (Binkofski et al., 1999a,b). Left BA44 incorporates the region commonly known as Broca's language area (Mohr, 1976). Experimental work demonstrates that humans activate their own appropriate motor regions at a sub-threshold level when they observe actions or hear words pertaining to actions (Fadiga et al., 2002; Fadiga and Craighero, 2004). It has been said that the human motor system resonates with observed external cues, with subthreshold activity in one's own motor system when viewing actions of another (Rizzolatti and Craighero, 2004; Etzel et al., 2008). The mirror neuron system (MNS) appears to be the biological motor component permitting "the first transition," one of acquiring "mimetic skill" required for the evolution of culture and cognition (Donald, 1993). Indeed, shortly after the initial experiments this claim was made in the seminal paper by Rizzolatti and Arbib (1998). The impact of the discovery of mirror neurons has been enormous, leading to a large debate with regard to social cognition as a whole (Keysers and Perrett, 2004; Gallese, 2007). Not least, it gave rise to studies to uncover the possible genetic changes that permitted this evolutionary leap (Corballis, 2004; Feuk et al., 2006), new means to interpret clinical disorders (Williams et al., 2001; Iacoboni and Mazziotta, 2007) and development of novel rehabilitation approaches for brain injury (Ertelt et al., 2007).

\section{REPLICATION OF BEHAVIOR FROM A MEMETIC PERSPECTIVE}

The term meme was first coined by Dawkins to describe cultural replicators and he described what have since become well worn examples, irritating pop tunes and ad jingles as replicators (Dawkins, 1976). Dawkins argued three pre-requisites for Darwinian evolution to occur, replication, variance, and selection and used the meme as an exemplifier that genes are not the "be all and end all" of evolution. Blackmore took the meme concept and expanded upon it. Suggesting memes as the second evolutionary force upon humans, specifically for the massive increase in brain size, drive for language acquisition, and culture as a whole (Blackmore, 1999). She argues that when humans acquired a key ability for imitation selective pressure occurred for those who could imitate best and thus most quickly acquire skills. Demonstrations of imitative ability would be in adornment, dance, song, and music, as well as in performance via hunting and gathering abilities. In her book, Blackmore argues that imitating gestures for communication would progress to more effective vocalizations for communication and that this would have led to language. Suddenly a new environment for replication emerged within the cognitive space of human brain activity and the physical world within which humans were able to interact. This process, was startlingly similar to that referred to as "mimesis" by Donald (1993) almost a decade earlier. Yet Donald did not focus on the evolutionary nature of memes, but on human capacities affected by "genetic" evolution. Memes, reproduce, have variance and undergo selection in a competitive environment (Cardoso and Atwell, 2010) and hence have all three pre-requisites (replication, variance, and selection) to a Darwinian evolutionary system. Consequently, memetic evolution constitutes a second evolutionary force affecting human development. These are very powerful claims and are confronted by a variety of objections (Boyd and Richardson, 2000; Dugatkin, 2000; Sperber, 2000) and with notable silence in the most pertinent area of current cognitive neuroscience research, the MNS (Iacoboni, 2005; Rizzolatti, 2005). The main reason, and perhaps a very good reason, for the reticence of neuroscience to join this debate is the lack of a working definition for a meme and its apparent impossibility to measure.

However, the point of interaction between the meme rich environment that we live in and our biological mass, or body, is of course the brain and therefore very much the stuff of cognitive neuroscience. Memes are proposed as being the primary driving force for the development of human intellect, and indirectly brain size after the key acquisition of imitative skills. Sometime between the appearance of the first stone tools created by Homo habilis ( 2.5 million years ago) and the arrival of Homo erectus (1.5 million years ago) our ancestors developed a considerably larger brain and more advanced mimetic culture (Donald, 1993). It is claimed that imitation conferred considerable advantage to imitators as they rapidly learned skills (fire making, hunting tactics) from others, circumventing less efficient trial and error approaches. Computer simulation of such a novel imitative phenotype moving within a population predicts logarithmic increases in learning ability, logarithmic increases in number of memes (imitated actions) per individual, and logarithmic increases in mean fitness (Higgs, 2000). 
This occurred independent of whether the behavior imitated was selected for its negative or positive effect. In other words, imitation as a novel strategy for learning predicts logarithmic increases in population fitness, independent of how well/badly judged its implementation by the newly endowed recipient of the skill. Moreover, this logarithmic change in evolution occurs, even when considerable negative consequences on fitness are considered, such as increased difficulties with childbirth due to increasing head size.

\section{WHAT EXACTLY IS A MEME? MEMES IN LAYMAN TERMS AND WITHIN THE FIELD OF MEMETICS}

Memes are replicants with the three pre-requisite properties for producing an evolutionary system, replication, variance, and selection. Memes replicate within the environment of human behavior (now technologically assisted) using human imitative behavior as their method for replication. To replicate, memes must pass through four key stages, assimilation (multimodal perception by an individual), retention (within memory), expression (by some motoric act, speech, or gesture, which can be perceived by others), and transmission (to another individual; Heylighen and Chielens, 2008). These terms are borrowed from genetics and are not altogether concordant with cognitive science's delineation of cognitive processes as will be discussed later. Memes have enormous variance made self evident by the heterogeneous cultural landscape observed across humanity. There is also considerable selection: a concept, language, or entire way of life that was here today can be gone tomorrow. According to Blackmore, a meme is not an innate behavior, conditioned response, emotion, or subjective experience. A meme is a behavior or set of behaviors (memeplex) which is learnt by observation and imitation. An individual word is a meme, as is a musical riff, a dance move, or a meaningful gesture. Religions, ideologies, and wider concepts, including the concept of the self, are proposed as "memeplexes" or groups of memes analogous to an organism (sets of genes replicate as single organisms, sets of memes may replicate as memeplexes). A meme is not how you feel when you dance or the innate pull toward your partner's lips, nor, according to Blackmore, the perceptual experience of seeing, smelling, or touching. This paper proposes a small change to these definitions.

The most common question regarding memes is, "what is a meme?" If an individual word is a meme, say for instance the word "chair" what in the world constitutes the "chair" meme? Does it include the neural substrates for perceiving a chair? Recognizing a chair? Knowing what to do with the chair? The neural substrates enabling one to pronounce the word chair? The neural substrates linked to your sense of current need for a chair? The neural substrates involved in understanding chair as component of the "seat" meme? The neural substrates linking a particular chair to a particularly nightmarish or orgasmic past experience? Is the actual chair itself a meme? Are the sound waves created by utterance of the word "chair" a meme? Is the image of a chair a meme? Is the written word "chair" a meme? When does the meme for "chair" become the meme for "seat?"

This paper attempts to offer a description of a meme in terms of biology, specifically neural connectivity profiles, but would be lacking without describing the memetic unit as currently described in information processing terms. The memetic unit has previously been usefully defined as a production rule with the form "if condition, then action" with actions leading to another condition or category, i.e., "if God then omnipotent," "if God then good," "if God punish bad." Sets of mutually reinforcing linked production rules (memes) could be combined to produce inferences and this set of rules would constitute a memeplex (for full explanation see, Heylighen and Chielens, 2008).

\section{A BREAKDOWN OF THE MEME IN BIOLOGICAL TERMS AND A WORKING HYPOTHESIS}

There are two types of questions above, first those that pertain to the internal transmission/storage of memes, within our CNS, and those that pertain to the external transmission/storage of memes in the world. These are consequently referred to as i-memes and e-memes respectively. Delineating e-memes may be relatively easy, even as they appear as conglomerate masses of many memes interwoven together. Humans go to considerable lengths to ensure the best fidelity of e-memes. This may be the reason behind copyright law, technological endeavors to ever increased fidelity of media reproduction, and the huge quantities of finance poured into the marketing and public relations branches of companies and political parties. It is via the media that the ever growing amount of memetic transmission occurs. The i-meme is of greater consequence however to cognitive neuroscience. The i-meme and e-meme are identical to that referred to as the memotype and mediotype by Heylighen and Chielens (2008).

Before presenting a working hypothesis however, it should be stated that the general point of this paper does not stand or fall on the issues of what constitutes an i-meme. Rather on whether changes to an i-meme can be measured directly by measuring changes in the connectivity profiles of brain regions as a result of changes to e-memes.

The i-meme constitutes the full set of "meme-specific" neural connections enabling e-memes to be perceived and transmitted by a communicative motor action in any one of its forms (verbal or gestural). Fundamentally, this neural network must incorporate the pre-requisite communicative motor component. Susan Blackmore states that memes are not perceptual experiences. The definition for the i-meme does not contradict this albeit let it be stressed that all i-memes will include (direct and/or indirect) connectivity between perceptual related brain regions and motor related brain regions. The i-meme is not "just" the neural substrates of perceptual experiences of an e-meme, it is the neural substrate of e-meme perception "linked to" the neural substrate of communicative motor act(s) required to reproduce that e-meme. The same could be said about emotional experiences. An i-meme cannot be solely an emotional experience yet an i-meme may contain an emotional component.

The primary components of the i-memes are envisaged as cortical and limbic. The pre-requisite motor component proposed is most likely to reside within the MNS. Previous studies demonstrate BA44 acts as a hub for initial integration of the multimodal aspects of new stimuli (Baumgaertner et al., 2007; Lahav et al., 2007; McNamara et al., 2008), essentially binding all aspects of an e-meme to the movement that signs for that meme (Figure 1).

Until proven otherwise one must include processes such as working memory, basal ganglia mediated learning, and other general cognitive processes into the neural network of an i-meme. It may well be that the regions underlying these processes are non-specifically recruited, but the experiments have not yet been conducted to eliminate these general brain functions from being included into a working definition of a meme. The basal 


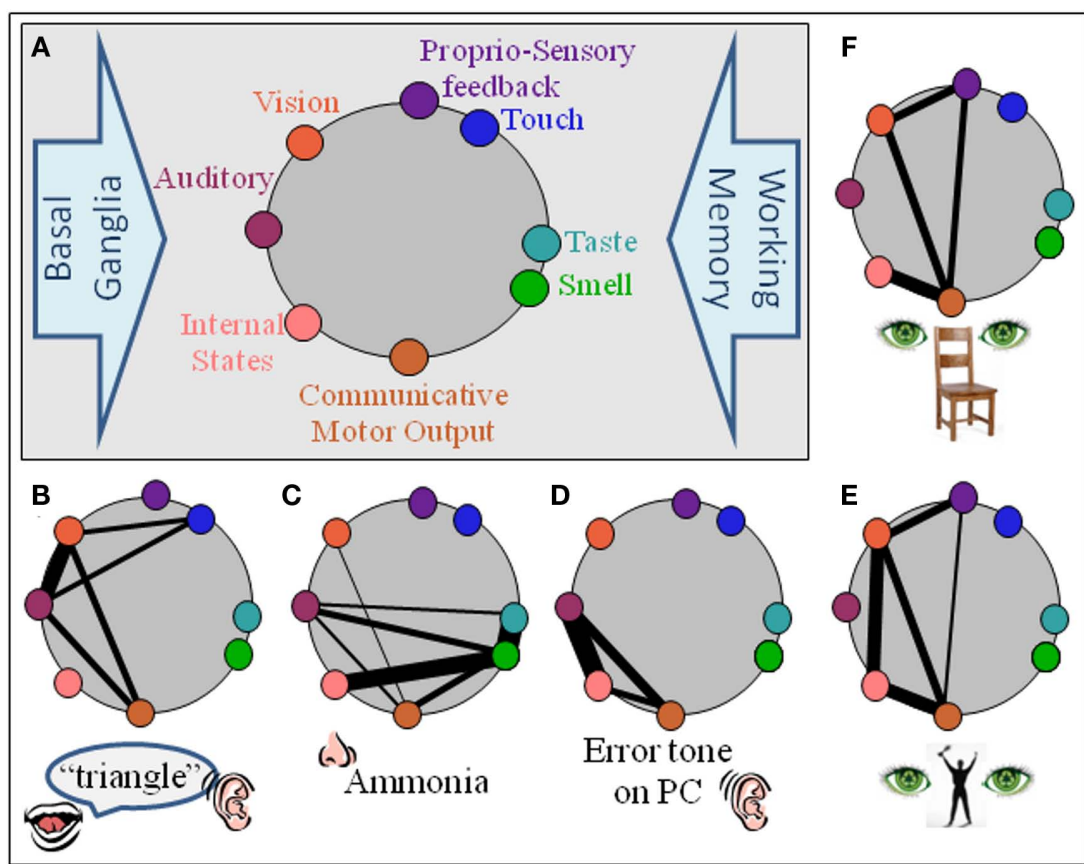

FIGURE 1 |An i-meme (internal representation of a meme) comprises the neural network which encodes it. (A) Basic structural components of non-requisite neural substrate architecture are auditory, vision, touch, propriosensory, taste, olfaction, and internal states (limbic). Communicative motor output is the neural substrate for the motor action required to communicate the meme and is the only pre-requisite component for a meme. All other components do not inherently require a representation within their domain. The basal ganglia (motor execution) and working memory are example processes unlikely to be part of an i-meme but to act upon them. (B-F) Possible neural substrate architectures for some exemplar i-memes, doorbell, ammonia, the irritating sound a personal computer makes when encountering an error, the gesture for victory. For simplicity, working memory and basal ganglia components are not incorporated. ganglia for example may provide controlled movement execution and behavioral reinforcement learning (Yelnik, 2008) across all i-memes.

One question above not answered by the i-meme/e-meme split is, where does the meme for "chair" become the meme for "seat?" The i-meme for these two concepts will have considerable functional and anatomical overlap, with shared neurons encoding the concept, yet the weights of the associations between connected neurons will be distinctly different. In this respect, they are specific. The anatomical localization for neural correlates of an i-meme is variable, function dependent, and context specific. The majority of memes would presumably not have specific-coding regions within primary visual cortex, but perhaps the meme known as "zebra" does"

\section{PRE-REOUISITES FOR MEMES}

Inherent requirements for function are useful delineators when mapping an evolutionary system. Memes evolve within the imitation capacity of the biological organism, primarily humans. The i-meme must be able to elicit behavior (expression) from its host which enables its transmission to other hosts. Therefore, to replicate, an i-meme must inherently have a communicative motor output (CMO). Every concept must inherently have a motor component, because to communicate (transmit), whether by speech, sign, or facial expression we must move our muscles (Donald, 2005; McNamara et al., 2008). Secondly, i-memes/concepts are not innate

${ }^{1}$ Primary visual cortex is particularly sensitive to basic visual stimuli such as black and white stripes. hardwired behaviors, they must initially be learned. Therefore, the i-meme replication machinery (the brain) must be able to rapidly link varied signals from any number of sensory modalities, internal states, and perhaps prior knowledge of similar concepts to the CMO (Figure 1). Economy and efficiency are fundamental principles of biology (Blake, 1992) and neural computation (Niven et al., 2007) with structure and function inextricably linked. The pre-requisite function of the replication system is the initial linkage of variable aspects of a concept. The pre-requisite structural component of an i-meme network is the brain region mediating the CMO. The most efficient means to integrate network-structure and function would be by co-localization of the two, i.e., an anatomical hub for integration of multimodal integration with the CMO. Human primary effectors, the hands and mouth produce the $\mathrm{CMO}$ and the neural circuits for complex hand and mouth actions are housed in the ventral-pre-motor cortex (Binkofski et al., 1999a,b). Therefore this brain region, one would deduce, would be the locus for mediating initial learning of novel i-memes. This region, also known as BA44, incorporating the speech center Broca's Areas is considered the human homolog to monkey region F5 and a key site of the MNS.

\section{CAN NEUROIMAGERS MEASURE MEMES? CONNECTIVITY ANALYSIS OF FMRI DATA}

Fascinating studies examining differing cognitive processes between cultures already demonstrate how language and culture may shape cognition as a whole (for review see Han and Northoff, 2008). For example, absolute and relative judgments, (either ignoring or taking 
into account context) in even a simple visual experiment showed strong effects of culturalization (Heddon et al., 2008). Such studies do not allow us to measure memes but provide a valuable resource for future experimental design.

To observe individual i-memes, we must measure brain activity changes over time in multiple regions of the brain simultaneously. Brain imaging technology, specifically functional magnetic resonance imaging ( $\mathrm{fMRI}$ ), has now advanced sufficiently for this to be achieved (Rogers et al., 2007; Guye et al., 2008; Rykhlevskaia et al., 2008). fMRI data allows quantification of the connectivity between different regions of the brain. Producing such profiles and observing changes in these profiles may be used to describe memetic processing at each stage of meme replication. A number of statistical approaches for assessing connectivity are now available for fMRI data of which any could theoretically be applied to studying memes. These include Pearson's correlation coefficients (Biswal et al., 1995), psychophysiological interaction (PPI) analysis (Friston et al., 1997), independent component analysis (McKeown et al., 1998), structural equation modeling (Gonzalez-Lima and McIntosh, 1994; McIntosh, 1998), dynamic causal modeling (Friston et al., 1993), Granger causality mapping (Goebel et al., 2003; Sato et al., 2006), and partial directed coherence (Baccalá and Sameshima, 2001; Sato et al., 2009).

Experiments observing the brain connectivity profile dynamics of i-memes as they are learnt and modulated over time can be envisaged. Such experiments would allow us to specifically describe where network activity changes as "meaning" of a concept changes. If a region changes activity as a consequence of changes in "meaning" of a meme then it is a component of the i-meme. If for example, basal ganglia can be shown to be only required for facilitating learning of memes in general, but not later recognition of memes then it can be excised as a component of memes. If memory systems can be shown to change connectivity profiles in relation to demands on memory and not to change as meanings change then memory components can be excised as a component of the working definition of an i-meme.

\section{DO WE MEASURE MEMES ALREADY?}

Many studies may already have unconsciously implemented individual i-meme formation as part of their study design. Gautier and Tarr (1997) trained subjects to become experts in identifying "greebles." Greebles are nonsense objects which could be classified first into families and then identified as individual greebles as a participant becomes more expert in identifying salient features. This study however did not measure during the learning stages of i-meme creation and did not assess the neural dynamics of what had been learnt. Koenig et al. (2005) measured brain activity as participants first learnt classification of novel animals named "crutters" using either rule based or similarity based strategy. This study did collect data during learning but did not assess neural dynamics. Deng et al. (2008) compared changes between "pre and post" learning of the semantic meaning associated with Chinese characters over time. All these studies and many other well conducted studies can be interpreted as studies in i-meme formation. Unfortunately they lack connectivity analysis which allows us to view the dynamics of memes. In the study conducted by McNamara et al. (2008) the dynamics of learning abstract, novel, sound-action associations was examined. The study was specifically designed as an exercise in creating artificial memes and measured the brain activity of that process. A PPI analysis (Friston et al., 1993) allowed functional connectivity profiles during learning of a novel meme to be measured. PPI essentially identifies brain regions which demonstrate an interaction between the BOLD activity over time from a seed region in the brain and a psychological variable, such as improved learning. The seed region is often localized by a standard univariate general linear model (GLM) analysis of the fMRI data or by a strong anatomical hypothesis. The two original variables (the time course and learning curve) are entered into a secondary univariate GLM analysis along with the interaction term. The output shows to what degree the data at each locus in the brain fits the model of increasing (or decreasing) connectivity between regions with increased learning. This approach does not allow for a de facto statement of directionality and only describes a functional connection, not an effective connection.

In the study, novel sounds were associated with novel gestures by viewing videos and having participants imitate the novel gesture. The participant's task was to learn the abstract sound-action association. These associations are akin to forming novel memes in that they create a concept (sound) which is assimilated into a gesture. The assimilation of the motor component is critical as otherwise the experiment is a pure associative learning experiment. The addition of requiring participants to imitate and generate a motor component transforms the stimuli into a concept that can be communicated, i.e., a meme. This creation of a simple, novel, abstract association which was assimilated into the motor cortex allowed for tracking of changes in neural activity and connectivity profiles between brain regions as learning and reproduction of an artificial meme was undertaken. Parametric decreases in activity were observed as a function of learning along with concomitant increases in functional connectivity (using PPI analysis) between many of these regions. Connectivity was observed between areas of the MNS and visual regions, between MNS and tonal processing regions, and MNS and working memory regions. Activity was also observed in regions involved in standard associative learning such as the hippocampus and caudate.

In summary, connectivity analyses tests whether there is any evidence to suggest regions showing activity during a task are interacting together as a network and crucially allows researchers to identify changes in these interactions over time. Even if the proposed definition of an i-meme is incorrect then it can still be asserted that the technology is available to measure the dynamics of i-memes as they evolve. Finally, even greater opportunity is now presented with the development of hyperscanning techniques (Montague et al., 2002). Hyperscanning is the running of two MRI machines with time-locked data acquisition and real time video link between the two participants. Using communicative paradigms future studies can measure correlations between two individual's brain activity during the process of meme transmission. Given such experimental protocols it may be found that a framework for understanding how ideas are transmitted and evolve between two people may become very useful.

Natural memes are highly complex, artificial memes are as complex as we choose to engineer them to be. In this sense, memes can be viewed as extremely compliant to experimental manipulation. As already demonstrated, there are sufficient neuroimaging methods for systematically exploring the processing and evolution of 
i-memes. Through tracing the development of artificial memes via the changes they make to neural activity, correlates to neural activity and behavior we can begin to delineate and define what may currently seem immeasurable.

\section{CONCLUDING REMARKS DOES COGNITIVE NEUROSCIENCE NEED MEMES?}

Perhaps memes are a red herring, a new set of terms for what is already studied. It could be argued that embracing terms such as "assimilation," "retention," "expression," and "transmission" as a step backward for cognitive science. After all, cognitive science has already a well defined taxonomy of cognitive skills to match these terms such as "learning," "memory," "action selection," and "perception." Learning for example is broken down into different types of learning, conditioned, procedural, etc. The molecular, neural, and systemic processes unpinning the learning function are further categorized. All the taxonomies are reductionist however and this is a double edged sword in terms of understanding a complex system such as cognition. As one reduces the component functions of a system then one becomes increasingly divorced from the overall function of the system. Learning and memory are cognitive functions but they are not "the function" of cognition. The sole function of cognition is to reproduce/replicate biologically and to reproduce behaviors that support reproduction. Viewing cognitive skills as part of a whole evolving "memetic system" allows us a framework in which to examine how these processes interact together to allow humans to function as single and social cognitive units.

A meme is not the same as a stimulus because the former describes the latter as part of a larger system. If a subject sees an abstract symbol with no meaning, it is a stimulus but not a meme. It is only a meme when the subject has learnt what the symbol signifies and how to communicate that concept to

${ }^{2} \mathrm{~A}$ meme must have passed through all four stages of replication, assimilation, retention, expression, and transmission, (Heylighen and Chielens, 2008) a stimulus must only be assimilated and retained.

\section{REFERENCES}

Arbib, M. A. (2005). From monkey-like action recognition to human language: an evolutionary framework for neurolinguistics. Behav. Brain Sci. 28, 105-124; discussion 125-167.

Baccalá, L. A., and Sameshima, K. (2001). PDC Partial directed coherence: a new concept in neural structure determination. Biol. Cybern. 84,463-474.

Baumgaertner, A., Buccino, G., Lange, R., McNamara, A., and Binkofski, F. (2007). Polymodal conceptual processing of human biological actions in the left inferior frontal lobe. Eur. J. Neurosci. 25, 881-889.

Berget, S. M., Moore, C., and Sharp, P. A. (1977). Spliced segments at the $5^{\prime}$ terminus of adenovirus 2 late mRNA. Proc. Natl. Acad. Sci. U.S.A. 74, 3171-3175.

Binkofski, F., and Buccino, G. (2006). The role of ventral premotor cortex in action execution and action understanding. J. Physiol. Paris 99, 396-405.

Binkofski, F., Buccino, G., Posse, S., Seitz, R. J., Rizzolatti, G., and Freund, H. (1999a). A fronto-parietal circuit for object manipulation in man: evidence from an fMRI-study. Eur. J. Neurosci. 11, 3276-3286.

Binkofski, F., Buccino, G., Stephan, K. M., Rizzolatti, G., Seitz, R. J., and Freund, H. J. (1999b). A parieto-premotor network for object manipulation: evidence from neuroimaging. Exp. Brain Res. 128, 210-213.

Biswal, B., Yetkin, F. Z., Haughton, V. M., and Hyde, J.S. (1995). Functional connectivity in the motor cortex of resting human brain using echo-planar MRI. Magn. Reson. Med. 34, 537-541.

Blackmore, S. (1999). The Meme Machine. Oxford: Oxford University Press.

Blackmore, S. (2005). "Evidence for memetic drive?" in Perspectives on Imitation, eds S. Hurley and N. Chater

someone else efficiently and accurately ${ }^{2}$. By utilizing such terms we can specify whether one is dealing with changing/evolving communicable stimuli (memes) or with static immutable percepts (stimuli). By separating the two concepts then one can begin to study differences between them, perhaps including the opportunity to disprove memes as useful at all. I predict that stimuli have extremely stable fMRI profiles even after being involved in considerable stimulus-stimulus associations with no pressure to communicate meaning. In contrast I predict i-memes will show far greater variability in fMRI profiles after an equal number of changes to their meaning (stimulus-stimulus associations plus need to communicate a symbolic meaning). Finally, let us assume for a moment that Blackmore is correct. Memes constitute the world we live in, natural and otherwise. The advertisements saturating our daily lives serve as "memelets," highly engineered and designed to maximize replication of the "brand" meme. Each word we utter is a successful meme, replicating as a useful part of our language. Entire sets of beliefs (memeplexes), i.e., political beliefs/behaviors such as democracy or Islamophobia (Iqbal, 2010), are proposed as large interrelated sets of memes that combine together to propagate more efficiently (Blackmore, 1999). It cannot be stated whether this is true or not without focusing on the pertinent question of what is a meme? Can it be measured? The importance of these questions is huge, far beyond that of an academic interest in how language came to be. The answers impact on current issues of high tension, social alienation in western societies, global ideological conflicts, media hegemony, and cultural homogenization in a media dominated globalized world. Cognitive neuroscience should focus on questions of how language and society is evolving "now" as well as how it genetically came into play in the first place. Utilizing modern communication devices such as mobile phone applications, social networking sites, closer work with social and evolutionary scientists, one can envisage longitudinal (ranging from hours to weeks) neuroimaging experiments measuring changes in connectivity between brain regions as a component process to cultural evolution.

(Cambridge, MA: Massachusetts Institute of Technology), 203-204.

Blake, R.W.(1992). Efficiencyand Economy in Animal Physiology. Cambridge: Cambridge University Press.

Boi, S., Solda, G., and Tenchini, M. L. (2004). Shedding light on the dark side of the genome: overlapping genes in higher eukaryotes. Curr. Genomics 5, 509-524.

Boyd, R., and Richardson, P. J. (2000). Meme theory oversimplifies how culture changes. Sci. Am. 283, 70-71.

Breathnach, R., Mandel, J. L., and Chambon, P. (1977). Ovalbumin gene is split in chicken DNA. Nature 270, 314-319.

Cardoso, G. C., and Atwell, J. W. (2010) Directional cultural change by modification and replacement of memes. Evolution 65, 295-300.

Cavara, N. A., and Hollmann, M. (2008). Shuffling the deck anew: how NR3 tweaks NMDA receptor function. $\mathrm{Mol}$. Neurobiol. 38, 16-26.
Corballis, M. C. (2004). FOXP2 and the mirror system. Trends Cogn. Sci. (Regul. Ed.) 8, 95-96.

Dawkins, R. (1976). The Selfish Gene. Oxford: Oxford University Press.

Deng, Y., Booth, J. R., Chou, T. L., Ding, G. S., and Peng, D. L. (2008). Item-specific and generalization effects on brain activation when learning Chinese characters. Neuropsychologia 46, 1864-1876.

Donald, M. (1993). Precis of origins of the modern mind and associated commentary. Behav. Brain Sci. 16, 737-791.

Donald, M. (2005). "Imitation and mimesis," in Perspectives on Imitation, eds S. Hurley and N. Chater (Cambridge, MA: Massachusetts Institute of Technology), 283-300.

Dugatkin, L. A. (2000). Animals imitate, too. Sci. Am. 283, 67.

Ertelt, D., Small, S., Solodkin, A., Dettmers, C., McNamara, A., Binkofski, F., and Buccino, G. (2007). Action observation has a positive impact on rehabili- 
tation of motor deficits after stroke. Neuroimage 36(Suppl.2), T164-T173.

Etzel, J. A., Gazzola, V., and Keysers, C. (2008). Testing simulation theory with cross-modal multivariate classification of fMRI data. PLoS ONE3, e3690. doi: 10.1371/journal.pone. 0003690

Fadiga, L., and Craighero, L. (2004). Electrophysiology of action representation. J. Clin. Neurophysiol. 21, 157-169.

Fadiga, L., Craighero, L., Buccino, G., and Rizzolatti, G. (2002). Speech listening specifically modulates the excitability of tongue muscles: a TMS study. Eur. J. Neurosci. 15, 399-402.

Ferrari, P. F., Rozzi, S., and Fogassi, L. (2005). Mirror neurons responding to observation of actions made with tools in monkey ventral premotor cortex. J. Cogn. Neurosci. 17, 212-226.

Feuk, L., Kalervo, A., Lipsanen-Nyman, M., Skaug, J., Nakabayashi, K., Finucane, B., Hartung, D., Innes, M., Kerem, B., Nowaczyk, M. J., Rivlin, J., Roberts, W., Senman, L., Summers, A., Szatmari, P., Wong, V., Vincent, J. B., Zeesman, S., Osborne, L. R., Cardy, J. O., Kere, J., Scherer, S. W., and Hannula-Jouppi, K. (2006). Absence of a paternally inherited FOXP2 gene in developmental verbal dyspraxia. Am. J. Hum. Genet. 79, 965-972.

Friston, K. J., Buechel, C., Fink, G. R., Morris, J., Rolls, E., and Dolan, R. J. (1997). Psychophysiological and modulatory interactions in neuroimaging. Neuroimage 6, 218-229.

Friston, K. J., Frith, C. D., Liddle, P. F., and Frackowiak, R. S. (1993). Functional connectivity: the principal-component analysis of large (PET) data sets. J. Cereb. Blood Flow Metab. 13, 5-14.

Gallese, V. (2007). Before and below "theory of mind": embodied simulation and the neural correlates of social cognition. Philos. Trans. R. Soc. Lond. B Biol. Sci. 362, 659-669.

Gallese, V., Fadiga, L., Fogassi., L., and Rizzolatti, G. (1996). Action recognition in the premotor cortex. Brain 119(Pt 2), 593-609.

Gautier, I., and Tarr, M. J. (1997). Becoming a "Greeble" expert: exploring mechanisms for face recognition. Vision Res. 37, 1673-1682.

Gilbert, W. (1978). Why genes in pieces? Nature 271, 501.

Goebel, R., Roebroeck, A., Kim, D. S., and Formisano, E. (2003). Investigating directed cortical interactions in time-resolved fMRI data using vector autoregressive modeling and Granger causality mapping. Magn. Reson. Imaging 21, 1251-1261.

Gonzalez-Lima, F., and McIntosh, A. R. (1994). Structural equation modeling and its application to network analysis in functional brain imaging. Hum. Brain Mapp. 1, 2-22.
Goodenough, O. R., and Dawkins, R. (1994). The 'St Jude' mind virus. Nature 371, 23-24.

Guye, M., Bartolomei, F., and Ranjeva, J.P. (2008). Imaging structural and functional connectivity: towards a unified definition of human brain organization? Curr. Opin. Neurol. 21,393-403.

Han, S., and Northoff, G. (2008). Culturesensitive neural substrates of human cognition: a transcultural neuroimaging approach. Nat. Rev. Neurosci. 9, 646-654.

Heddon, T., Ketay, S., Aron, A., Markus, H. R., and Gabrieli, J. D. (2008). Cultural influences on neural substrates of attentional control. Psychol. Sci. 19, 12-17.

Heylighen, F., and Chielens, K. (2008). "Evolution of Culture, Memetics," in Encyclopedia of Complexity and Systems Science, ed. B. Meyers (Vrije Universiteit Brussel, Brussel: Springer).

Higgs, P. G. (2000). The mimetic transition: a simulation study of the evolution of learning by imitation. Proc. Biol. Sci. 267, 1355-1361.

Iacoboni, M. (2005). "Understanding others: imitation, language, and empathy,' in Perspectives on Imitation, S. Hurleyand N. Chater (Cambridge, MA: Massachusetts Institute of Technology), 77-100.

Iacoboni,M.,andMazziotta,J.C.(2007).Mirror neuron system: basic findings and clinical applications. Ann. Neurol 62, 213-218.

Iqbal, Z. (2010). Understanding Islamophobia: conceptualizing and measuring the construct. Eur. J. Social Sci. 13, 574-590.

Iriki, A. (2006). The neural origins and implications of imitation, mirror neurons and tool use. Curr. Opin. Neurobiol. 16, 660-667.

Keysers, C., and Perrett, D. I. (2004). Demystifying social cognition: a Hebbian perspective. Trends Cogn. Sci. (Regul. Ed.) 8, 501-507.

Koenig, P., Smith, E. E., Glosser, G., DeVita, C., Moore, P., McMillan, C., Gee, J., and Grossman, M. (2005). The neural basis for novel semantic categorization. Neuroimage 24, 369-383.

Kohler, E., Keysers, C., Umilta, M. A., Fogassi, L., Gallese, V., and Rizzolatti, G. (2002). Hearing sounds, understanding actions: action representation in mirror neurons. Science 297, 846-848.

Lahav, A., Saltzman, E., and Schlaug, G. (2007). Action representation of sound: audiomotor recognition network while listening to newly acquired actions. J. Neurosci. 27, 308-314.

Lynch, K. W. (2004). Consequences of regulated pre-mRNA splicing in the immune system. Nat. Rev. Immunol. 4, 931-940.

McIntosh, A. R. (1998). Understanding neural interactions in learning and memory using functional neuroimaging. Ann. N. Y.Acad. Sci. 855, 556-571.

McKeown, M. J., Makeig, S., Brown, G. G., Jung, T. P., Kindermann, S. S., Bell, A.
J., and Sejnowski, T. J. (1998). Analysis of fMRI data by blind separation into independent spatial components. Hum. Brain Mapp. 6, 160-188.

McNamara, A., Buccino, G., Wolbers, T., Glaescher, J., Baumgaertner, A., and Binkofski, F. (2008). Neural dynamics of learning sound-action associations in the mirror neuron system. PLoS ONE 3, e3845. doi: 10.1371/journal. pone. 0003845

Mendel, G. (1951). FACSIMILE of Gregor Mendel's "experiments in plant hybridization". J. Hered. 42, 3-47.

Mohr,J.P. (1976). "Broca's area and Broca's aphasia," in Studies in Neurolinguistics, eds H. Witaker and N. A. Witater (New York: Academic Press), 201-235.

Montague, P. R., Berns, G. S., Cohen, J. D., McClure, S. M., Pagnoni, G., Dhamala, M., Wiest, M. C., Karpov, I., King, R. D., Apple, N., and Fisher, R. E. (2002). Hyperscanning: simultaneous fMRI during linked social interactions. Neuroimage 16, 1159-1164.

Niven, J.E., Anderson, J.C., and Laughlin, S. B. (2007). Fly photoreceptors demonstrate energy-information tradeoffs in neural coding. PLoS Biol. 5, e16. doi: 10.1371/journal.pbio.0050116

Normark, S., Bergstrom, S., Edlund, T., Grundstrom, T., Jaurin, B., Lindberg, F. P., and Olsson, O. (1983). Overlapping genes. Annu. Rev. Genet. 17, 499-525.

Raos, V., Umilta, M. A., Gallese, V., and Fogassi, L. (2004). Functional properties of grasping-related neurons in the dorsal premotor area $\mathrm{F} 2$ of the macaque monkey. J. Neurophysiol. 92, 1990-2002.

Raos, V.,Umilta,M.A.,Murata,A.,Fogassi,L. and Gallese, V. (2006). Functional properties of grasping-related neurons in the ventral premotor area 55 of the macaque monkey. J. Neurophysiol. 95, 709-729.

Rizzolatti, G. (2005). “The mirror neuron system and imitation," in Perspectives on Imitation, eds S. Hurley and N. Chater (Cambridge, MA: Massachusetts Institute of Technology), 55-76.

Rizzolatti, G., and Arbib, M. A. (1998) Language within our grasp. Trends Neurosci. 21, 188-194.

Rizzolatti, G., and Craighero, L. (2004). The mirror-neuron system. Annu. Rev Neurosci. 27, 169-192.

Rizzolatti, G., Fadiga, L., Gallese, V., and Fogassi, L. (1996). Premotor cortex and the recognition of motor actions. Brain Res. Cogn. Brain Res. 3, 131-141.

Rogers, B.P.,Morgan, V.L.,Newton,A.T., and Gore, J. C. (2007). Assessing functional connectivityin the human brain by fMRI Magn. Reson. Imaging 25, 1347-1357.

Roth, G., and Dicke, U. (2005). Evolution of the brain and intelligence. Trend Cogn. Sci. (Regul. Ed.) 9, 250-257.

Rykhlevskaia, E., Gratton, G., and Fabiani, M. (2008). Combining structural and functional neuroimaging data for studying brain connectivity: a review. Psychophysiology 45, 173-187.

Sanna,C.R.,Li,W.H., and Zhang,L. (2008), Overlapping genes in the human and mouse genomes. BMC Genomics 9, 169. doi: 10.1186/1471-2164-9-169

Sato, J. R., Junior, E. A., Takahashi, D. Y., de Maria Felix, M., Brammer, M. J., and Morettin, P. A. (2006). A method to produce evolving functional connectivity maps during the course of an fMRI experiment using waveletbased time-varying Granger causality. Neuroimage 31, 187-196.

Sato, J. R., Takahashi, D. Y., Arcuri, S. M., Sameshima, K., Morettin, P. A., and Baccalá,L.A. (2009). Frequency domain connectivity identification: an application of partial directed coherence in fMRI. Hum. Brain. Mapp. 30, 452-461.

Sperber, D. (2000). "An objection to the memetic approach to culture," in Darwinizing Culture: The Status of Memetics as a Science, ed. R. Aunger (Oxford, NY: Oxford University Press), 163-175.

Stumpf, M. P., Thorne, T., de Silva, E., Stewart, R., An, H. J., Lappe, M., and Wiuf, C. (2008). Estimating the size of the human interactome. Proc. Natl. Acad. Sci. U.S.A. 105, 6959-6964.

Umiltà, M. A., Kohler, E., Gallese, V., Fogassi, L., Fadiga, L., Keysers, C., and Rizzolatti, G. (2001). I know what you are doing. A neurophysiological study. Neuron 31, 155-165.

Veeramachaneni, V., Makalowski, W., Galdzicki, M., Sood, R., and Makalowska, I. (2004). Mammalian overlapping genes: the comparative perspective. Genome Res. 14, 280-286. Williams, J. H., Whiten, A., Suddendorf, T., and Perrett, D. I. (2001). Imitation, mirror neurons and autism. Neurosci. Biobehav. Rev. 25, 287-295.

Yelnik, J. (2008). Modeling the organization of the basal ganglia. Rev. Neurol. (Paris) 164, 969-976.

Conflict of Interest Statement: The author declares that the research was conducted in the absence of any commercial or financial relationships that could be construed as a potential conflict of interest.

Received:22 December 2010; paperpending published: 24 January 2011; accepted: 12 May 2011; published online: 25 May 2011. Citation: McNamara A (2011) Can we measure memes? Front. Evol. Neurosci. 3:1. doi: 10.3389/fnevo.2011.00001 Copyright () 2011 McNamara. This is an open-access article subject to a nonexclusive license between the authors and Frontiers MediaSA, which permits use, distribution and reproduction in other forums, provided the original authors and source are credited and other Frontiers conditions are complied with. 